\title{
Fatal or not? Finding errors that lead to dialogue breakdowns in chat-oriented dialogue systems
}

\author{
Ryuichiro Higashinaka ${ }^{1}$, Masahiro Mizukami ${ }^{2}$, Kotaro Funakoshi ${ }^{3}$, \\ Masahiro Araki $^{4}$, Hiroshi Tsukahara $^{5}$, Yuka Kobayashi ${ }^{6}$ \\ ${ }^{1}$ NTT Corporation, ${ }^{2}$ Nara Institute of Science and Technology \\ ${ }^{3}$ Honda Research Institute Japan, ${ }^{4}$ Kyoto Institute of Technology \\ ${ }^{5}$ Denso IT Laboratory, Inc., ${ }^{6}$ Toshiba Corporation
}

\begin{abstract}
This paper aims to find errors that lead to dialogue breakdowns in chat-oriented dialogue systems. We collected chat dialogue data, annotated them with dialogue breakdown labels, and collected comments describing the error that led to the breakdown. By mining the comments, we first identified error types. Then, we calculated the correlation between an error type and the degree of dialogue breakdown it incurred, quantifying its impact on dialogue breakdown. This is the first study to quantitatively analyze error types and their effect in chat-oriented dialogue systems.
\end{abstract}

\section{Introduction}

Chat-oriented or open-domain dialogue systems have recently been attracting attention from social and entertainment aspects (Bickmore and Cassell, 2001; Banchs and Li, 2012; Wilcock and Jokinen, 2013). However, since they need to deal with open-domain utterances, which current natural language processing techniques are not mature enough to handle appropriately, the system inevitably makes errors. This discourages users from talking to the system, leading to dialogue breakdowns in conversation (Martinovsky and Traum, 2003). Here, dialogue breakdowns denote points in dialogue where users are unable to continue the conversation.

This paper aims to find errors that lead to dialogue breakdowns in chat-oriented dialogue systems. Our approach is two-fold: (1) identify error types in chat-oriented dialogue systems, and (2) calculate their impact on dialogue breakdown. For (1), we first collect chat dialogues between an automated system and users, annotate the dialogues with dialogue breakdown labels, and collect comments that describe the error that led to the break- downs. After that, we apply automatic clustering methods to the comments to obtain clusters of comments, each of which corresponds to a particular error type. For (2), we calculate the correlation between an error type and the degree of dialogue breakdown it incurs. This way, we can quantify the impact of an error type on dialogue breakdown. By our approach, we hope to distinguish fatal errors from non-fatal ones, providing useful guidelines for dialogue system developers to avoid breakdowns in chat-oriented dialogue systems.

\section{Data Collection}

For data collection, we asked dialogue researchers and their collaborators in Japan to use our chatoriented dialogue system. The system is textbased and enables chatting in Japanese. It was built by wrapping a chat API provided by NTT DOCOMO (Onishi and Yoshimura, 2014). Since the API works on the basis of (Higashinaka et al., 2014), which uses a number of natural language processing techniques to understand and generate utterances, we expected to obtain a wide variety of dialogues, and hence, a variety of errors.

A total of 116 users chatted with the system, resulting in 1,146 dialogues. Here, each dialogue was controlled to be 21 utterances long: one system prompt with ten utterances each from the user and system. Then, we randomly sampled 100 dialogues (called the init100 data set) for dialogue breakdown annotation. Twenty-four annotators subjectively labeled each system utterance in init 100 with one of the following three dialogue breakdown labels:

(NB) Not a breakdown: The conversation is easy to continue after the system utterance in question.

(PB) Possible breakdown: The conversation is difficult to continue smoothly after the system utterance in question. 


\begin{tabular}{|c|c|c|c|c|c|c|}
\hline & Utterance & NB & PB & B & $\mathrm{C}$ & Comments \\
\hline S0 & Hello! Talk to me anytime. & 24 & 0 & 0 & 0 & \\
\hline $\mathrm{U} 1$ & It's hot today, isn't it? & & & & & \\
\hline S1 & $\begin{array}{l}\text { Please tell me your favorite movie } \\
\text { genre. }\end{array}$ & 20 & 2 & 2 & 2 & $\begin{array}{l}\text { User utterance is not considered. / The system ig- } \\
\text { nored user's intended topic. }\end{array}$ \\
\hline $\mathrm{U} 2$ & I like action movies. & & & & & \\
\hline S2 & Action movies, I like them. & 21 & 3 & 0 & 0 & \\
\hline U3 & What kind of movies do you like? & & & & & \\
\hline S3 & I like Japanese ones. & 23 & 1 & 0 & 0 & \\
\hline $\mathrm{U} 4$ & That's great. & & & & & \\
\hline S4 & Thank you. & 24 & 0 & 0 & 0 & \\
\hline U5 & $\begin{array}{l}\text { Do you know what movie will be } \\
\text { aired on Friday night? }\end{array}$ & & & & & \\
\hline S5 & Yes, yes. & 4 & 11 & 9 & 4 & $\begin{array}{l}\text { The system did not mention a movie name. / It } \\
\text { answered only "yes" but without saying a movie } \\
\text { name. / This is not an answer to the question. / } \\
\text { The conversation is not valid. }\end{array}$ \\
\hline U6 & I haven't heard of that movie. & & & & & \\
\hline S6 & That's good. & 3 & 10 & 11 & 4 & $\begin{array}{l}\text { This is not a response to a question. / Strange an- } \\
\text { swer. / It is not clear what is good. / The conversa- } \\
\text { tion is not valid. }\end{array}$ \\
\hline U7 & Oh, is it good? I will watch it then. & & & & & \\
\hline S7 & That's good! & 18 & 3 & 3 & 1 & It is not clear what is good. \\
\hline U8 & $\begin{array}{l}\text { Recently, I have had lots of free } \\
\text { time. }\end{array}$ & & & & & \\
\hline S8 & That's great. & 15 & 7 & 2 & 0 & \\
\hline U9 & Are you busy? & & & & & \\
\hline S9 & Indeed so. & 24 & 0 & 0 & 0 & \\
\hline U10 & Take care. Have some rest. & & & & & \\
\hline S10 & I see. & 10 & 9 & 5 & 2 & The system should thank the user. / Strange answer. \\
\hline
\end{tabular}

Figure 1: Example dialogue with the number of breakdown labels for each system utterance. $\mathrm{S}$ and $\mathrm{U}$ stand for system and user utterances, respectively. $\mathrm{C}$ stands for the number of comments provided. The dialogue was originally in Japanese and was translated by the authors.

(B) Breakdown: The conversation is difficult to continue at all after the system utterance in question.

The annotators were instructed to provide voluntary comments to describe the errors that led to the breakdowns. Table 1 shows the statistics of the dialogue breakdown labels and comments. Figure 1 shows an example dialogue with the number of breakdown labels and comments for each system utterance. In this example, S5 and S6 were annotated with nine and eleven breakdown labels, respectively, both having four comments.

The inter-annotator agreement of dialogue breakdown annotation in Fleiss' kappa was 0.276, which seems relatively low. One reason for this is obviously the subjective nature of the task. Another possible reason is that we intentionally did not set rigid guidelines for dialogue breakdown annotation so as to explore possible error types in chat-oriented dialogue systems. When we merge $\mathrm{PB}$ and $\mathrm{B}$ and make it a two-class annotation, the agreement becomes 0.396 (moderate agreement), showing that the subjects share some common conception about dialogue breakdown.

\begin{tabular}{c|r|r} 
Breakdown label & \# of labels & \# of comments \\
\hline NB & 14,212 & 57 \\
PB & 5,322 & 1,818 \\
B & 4,466 & 1,511 \\
\hline
\end{tabular}

Table 1: Statistics related to breakdown labels and comments in init100 data set. Note that NB also had some opinions as comments.

\section{Analysis}

\subsection{Automatic clustering of comments}

We first need to identify the error types in chatoriented dialogue systems. For this, we applied an automatic clustering method to the comments to obtain clusters of comments. Our idea is that, since each comment describes a particular error that led to a breakdown, a cluster of comments is likely to represent an error type. Since the number of clusters is difficult to know in advance, we turn to a non-parametric Bayesian method called the Chinese restaurant process (CRP) as a clustering method. CRP can infer the number of clusters automatically from data (Pitman, 1995).

We applied CRP to the 1,511 comments given 


\begin{tabular}{r|r|l|l} 
ID & Size & Interpretation & Representative words in the cluster \\
\hline 2 & 259 & General quality & dialogue, well-formed, consideration, conversation \\
0 & 194 & Not understandable & understand, meaning, what \\
6 & 148 & Ignore user utterance & ignore, user, utterance \\
7 & 134 & Ignore user question & answer, question, partner, respond \\
1 & 113 & Unclear intention & unclear, intention, meaning, utterance \\
8 & 107 & Contradiction & doubtful, change, negative (as opposed to positive), previous \\
16 & 100 & Analysis failure & analysis, recognition, related, understand \\
4 & 95 & Inappropriate answer & response, inappropriate, invalid, answer \\
5 & 77 & Repetition & say, add, mind, strange, tired \\
3 & 54 & Grammatical error & (words specific to particular grammar usage) \\
14 & 53 & Expression error & (words specific to particular expressions) \\
11 & 39 & Topic-change error & change, topic, sudden, mismatch, response \\
12 & 38 & Violation of common sense & match, flow, common sense, against, connection \\
13 & 36 & Word usage error & (words specific to particular word usage) \\
10 & 35 & Diversion & story, different \\
15 & 25 & Mismatch in conversation & match, story \\
9 & 4 & Social error & (no words) \\
\hline
\end{tabular}

Table 2: Clusters by CRP of 1,511 comments given to breakdowns. The clusters are ordered by size.

to breakdown labels (B labels). For the clustering, we used the same procedure as (Higashinaka et al., 2011); each datum (comment) was represented by a word frequency vector, and the probability that it belonged to a particular cluster was calculated by using the likelihood that the words are generated from the word distribution of that cluster. The hyper-parameters $\alpha$ and $\beta$ were both set to 0.1 and the number of iterations for Gibbs sampling was 10,000. See (Higashinaka et al., 2011) for the details of the procedure.

Table 2 shows the clustering results. We obtained 17 clusters. For each cluster, we mined representative words by a log-likelihood ratio test, which uses a two-by-two matrix to test the independence of a word to a particular cluster. By looking at the representative words and also the raw comments, we came up with the interpretations of the clusters as indicated in the table. Although we do not go into the details of the clusters one by one, each cluster seems to successfully represent a certain error type in chat-oriented dialogue systems. We also applied CRP to the 3,329 comments given to PB and B to obtain similar clusters except that we additionally had clusters whose interpretations are as follows: inability to handle invalid user input, missing topic, missing information, mismatch in response, no reaction, and no information. They account for about $13.3 \%$ of the comments and mostly concern missing elements (such as missing arguments) in dia- logue. Since such missing elements can be complemented by follow-on utterances in dialogue, they only appear in the comments for PB; they do not lead to an immediate dialogue breakdown.

To further categorize the clusters, we applied a hierarchical clustering (an agglomerative clustering) to the clusters. Here, a cluster was represented by the word frequency vector of all comments contained in the cluster, and the similarity of the clusters was calculated by cosine similarity of word frequency vectors. For the linkage criterion, we used Ward's method. Figure 2 shows the clustering results. The figure indicates that there are the following eight main error categories (E1-E8):

(E1) Clusters 2 and 16 concern the general ability of a system.

(E2) Clusters 7, 5, and 8 relate to context awareness: the ability to recognize when it is asked a question and to recognize what has been said before.

(E3) Clusters 13, 3, and 14 concern the language generation (surface realization) ability.

(E4) Clusters 4 and 6 concern the response ability: the ability to answer questions and to create utterances relevant to the previous user utterance.

(E5) Cluster 1 relates to the exhibition of an intention or a plan: the ability to make clear the 


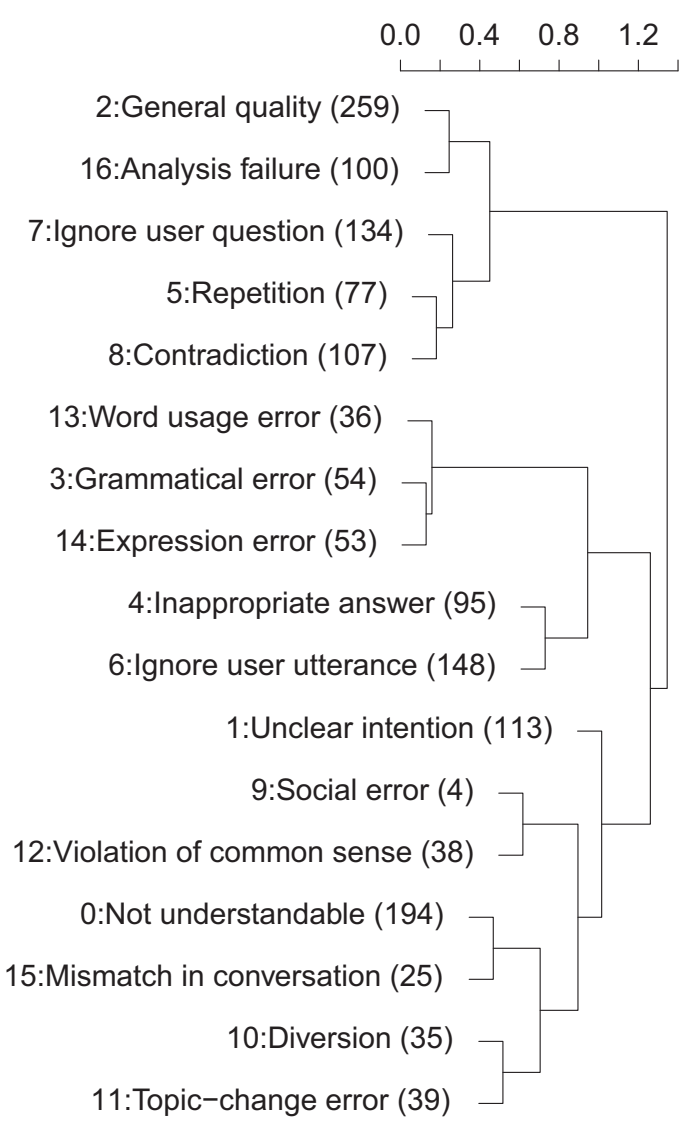

Figure 2: Hierarchical clustering applied to the obtained clusters. The numbers in parentheses denote cluster size.

purpose of an utterance.

(E6) Clusters 9 and 12 relate to the social ability: the ability not to offend users or say things that are not socially acceptable.

(E7) Clusters 0 and 15 concern the understandability of an utterance: the ability to generate utterances that have clear meanings in the context of the conversation.

(E8) Clusters 10 and 11 relate to the awareness of current topics.

\subsection{Analyzing the impact of error types}

Having identified the error types and error categories, we investigated their impact on dialogue breakdown. For this purpose, we examined the correlation between an error type and its degree of breakdown: the higher the correlation, the more it is related to dialogue breakdown. Specifically, we calculated the correlation ratio $(\eta)$ between the existence of a comment belonging to a particular cluster (error type) and the number of breakdown labels (B labels). Note that the correlation ratio

\begin{tabular}{r|l|cc} 
ID & Interpretation & Cat & $\eta$ \\
\hline 0 & Not understandable & E7 & 0.38 \\
7 & Ignore user question & E2 & 0.37 \\
2 & General quality & E1 & 0.36 \\
1 & Unclear intention & E5 & 0.36 \\
6 & Ignore user utterance & E4 & 0.24 \\
13 & Word usage error & E3 & 0.18 \\
16 & Analysis failure & E1 & 0.17 \\
4 & Inappropriate answer & E4 & 0.17 \\
3 & Grammatical error & E3 & 0.15 \\
12 & Violation of common sense & E6 & 0.15 \\
8 & Contradiction & E2 & 0.14 \\
5 & Repetition & E2 & 0.11 \\
9 & Social error & E6 & 0.10 \\
10 & Diversion & E8 & 0.09 \\
11 & Topic-change error & E8 & 0.06 \\
15 & Mismatch in conversation & E7 & 0.06 \\
14 & Expression error & E3 & 0.02 \\
\hline
\end{tabular}

Table 3: Correlation ratio $(\eta)$ between the existence of a comment of a cluster (error type) and the number of breakdown labels. "Cat" denotes the error category of an error type.

is equivalent to Pearson's correlation coefficient except that it can be applied to categorical data. The $\eta$ ranges from 0 to 1 . For calculation, we first extracted data that had one or more B labels and one or more corresponding comments (we had 556 such samples in our data). Then, we calculated the correlation ratios.

Table 3 shows the correlation ratios for the error types. Clearly, not all error types have the same level of correlation. At the top of the table, there are four salient error types with similar $\eta$ values: "Not understandable", "Ignore user question", "General quality", and "Unclear intention". Putting aside "General quality", which seems to concern the overall dialogue ability, the error types that we need to consider as fatal would seem to be the other three. Other errors seem to be less important with lower $\eta$ values. "Expression error", which concerns the use of unnatural expressions, was found the least important.

When we look at the error categories, we can see an interesting result that it is NOT the error category that determines the fatality of errors but the specificity of error types. For example, "Not understandable" and "Mismatch in conversation" are both under error category E7 but have totally different effects on perceived breakdown. The same can be said for error types in E2. 
Note that, although the values of correlation ratio seem rather low, the correlation often becomes low when it comes to subjective judgments (Higashinaka et al., 2004). Considering that we deal with chat-oriented dialogues, which are less restricted than task-oriented ones, we consider the current values of correlation ratio to be acceptable. Here, the important finding is that several error types are comparatively more important than the others.

\section{Related work}

Few studies have analyzed breakdowns in conversation. One exception is the study by Martinovsky and Traum (2003), who discussed possible causes of breakdowns they observed. Our work is different in that we systematically identify error types and quantitatively evaluate their effect. Our work can be seen as listing up errors in dialogue systems. A number of studies have aimed to create a taxonomy of errors (Bernsen et al., 1996; Möller, 2002; Paek, 2003), but their taxonomies are created manually and focus on task-oriented dialogue systems.

\section{Summary and future work}

By processing dialogue data with dialogue breakdown annotations and comments, this paper identified 17 error types that can be further categorized into eight error categories. By calculating correlation ratios, we discovered three error types that can be fatal: "Not understandable", "Ignore user question", and "Unclear intention". To avoid dialogue breakdowns, it is suggested that we need to make clear the meanings of system utterances, not ignore user questions, and show some intention behind system utterances. The findings will be useful for dialogue system developers who want to realize smooth human-machine interaction in chat-oriented dialogue systems and possibly in dialogue design as a whole.

For future work, we plan to consider ways to improve systems on the basis of our findings and also verify the generality of the results on data using other systems. To accurately detect dialogue breakdowns, dialogue systems researchers will need to collaborate. To this end, we are planning to organize an evaluation workshop on dialogue breakdown detection. For use in the evaluation workshop as well as in dialogue research in general, we have released our data with all the annotations and comments to the public. ${ }^{1}$

\section{Acknowledgments}

The "Project Next NLP" project in Japan is addressing the problems related to analyzing errors in natural language processing systems. One subgroup, comprising more than 32 researchers from 15 institutions, is collaborating in the performance of a dialogue task. We thank the members of this sub-group for the data collection, annotation, and fruitful discussions. We also thank NTT DOCOMO for letting us use their chat API for data collection.

\section{References}

Rafael E Banchs and Haizhou Li. 2012. IRIS: a chatoriented dialogue system based on the vector space model. In Proc. the ACL 2012 System Demonstrations, pages 37-42.

Niels Ole Bernsen, Hans Dybkjaer, and Laila Dybkjaer. 1996. Principles for the design of cooperative spoken human-machine dialogue. In Proc. ICSLP, volume 2, pages 729-732.

Timothy W. Bickmore and Justine Cassell. 2001 Relational agents: a model and implementation of building user trust. In Proc. CHI, pages 396-403.

Ryuichiro Higashinaka, Noboru Miyazaki, Mikio Nakano, and Kiyoaki Aikawa. 2004. Evaluating discourse understanding in spoken dialogue systems. ACM Transactions on Speech and Language Processing (TSLP), 1:1-20.

Ryuichiro Higashinaka, Noriaki Kawamae, Kugatsu Sadamitsu, Yasuhiro Minami, Toyomi Meguro, Kohji Dohsaka, and Hirohito Inagaki. 2011. Unsupervised clustering of utterances using non-parametric bayesian methods. In Proc. INTERSPEECH, pages 2081-2084.

Ryuichiro Higashinaka, Kenji Imamura, Toyomi Meguro, Chiaki Miyazaki, Nozomi Kobayashi, Hiroaki Sugiyama, Toru Hirano, Toshiro Makino, and Yoshihiro Matsuo. 2014. Towards an open-domain conversational system fully based on natural language processing. In Proc. COLING, pages 928-939.

Bilyana Martinovsky and David Traum. 2003. The error is the clue: Breakdown in human-machine interaction. In Proc. ISCA Workshop on Error Handling in Spoken Dialogue Systems, pages 11-16.

Sebastian Möller. 2002. A new taxonomy for the quality of telephone services based on spoken dialogue systems. In Proc. SIGDIAL, pages 142-153.

${ }^{1}$ https://sites.google.com/site/ dialoguebreakdowndetection/ 
Kanako Onishi and Takeshi Yoshimura. 2014. Casual conversation technology achieving natural dialog with computers. NTT DOCOMO Technical Jouranl, 15(4):16-21.

Tim Paek. 2003. Toward a taxonomy of communication errors. In Proc. ISCA Workshop on Error Handling in Spoken Dialogue Systems, pages 53-58.

Jim Pitman. 1995. Exchangeable and partially exchangeable random partitions. Probability theory and related fields, 102(2):145-158.

Graham Wilcock and Kristiina Jokinen. 2013. Wikitalk human-robot interactions. In Proc. ICMI, pages 73-74. 